\title{
Does the Engram of Kindling Model the Engram of Normal Long Term Memory?
}

\author{
G. V. GODDARD AND R. M. DOUGLAS
}

SUMMARY: The kindling effect is a relatively permanent alteration in brain function which results from repeated electrical or chemical stimulation and culminates in the appearance of electrographic and behavioral convulsions whenever the original stimulus is reapplied. The effect results from tetanic activation in the anterior cortex, limbic system or associated areas of the adult mammalian brain, and the lasting alterations are transynaptic and quite widespread. They are based in part on synaptic facilitation, and they are accompanied by specific alterations in normal behavior. In these and other respects, kindling is analogous to normal learning. It is possible that the stored component (engram) of kindling involves the same physiological mechanism as the engram of normal long term memory. Morphological study of identified synapses has not provided conclusive evidence for an anatomical substrate of kindling, but physiological experiments demonstrate a lasting potentiation of the excitatory post-synaptic potential.

RÉSUMÉ: Les auteurs soulignent la similarité entre le phénomène du "kindling" et celui de l'apprentissage. Il est possible que l'engramme du "kindling" utilise les mêmes mécanismes physiologiques que l'engramme de la mémoire à long terme normale. Les études anatomiques n'ont pas encore identifie de modifications synaptiques, mais les expériences physiologiques indiquent une potentiation durable $d u$ potentiel excitatoire post-synaptique.

From the Department of Psychology, Dalhousie University, Halifax.

Reprint request to Dr. G. V. Goddard, Dept. of Psychology, Dalhousie University, Halifax, Nova Scotia, B3H 4JI Canada.

This research was supported by the National Research Council of Canada.

We wish to thank Dr. R. S. Rodger for providing extensive assistance with our statistical analyses of synaptic morphology.
The term "engram" has been used by Lashly (1950) and others to indicate something which is stored in the brain to represent a memory. Although temporary engrams would exist for short term memories, we are concerned here with the engram of long term memory. It is a theoretical construction which implies some tangible substrate for the encoding and storage of experience.

Theories of learning from Descartes to modern times (Hebb, 1949; Marr, 1969; Milner, 1957) have typically specified assumptions regarding the form or location of the engram, but empirical evidence has been lacking. One method of obtaining evidence would be to create an artificial alteration in brain function at some convenient and specific location and examine the details and conditions surrounding this artificial engram.

Before such an artificial engram can be accepted as a reasonable test case, it must be shown to satisfy a number of minimal conditions. That is, any finding which pretends to model the engram must be a change in neural processing which can result from neural activation and which is relatively permanent. It must be a selective change in the sense that it provides, theoretically, the possibility of one memory being distinct from another, and be a nondegenerative change in the sense that the acquisition of new memories should not necessarily delete innate or previously well learned behavior patterns. By preference it should be demonstrated in a mammalian brain, but certainly it should be demonstrated in the adult animal since adult learning may not always be the same as differential development in infants. When it occurs, the change must be able to alter normal behavior in a lasting way, and it must be a change that can be induced by environmental sensory input. In addition, it is desirable to show that the phenomenon has other similarities to the more salient aspects of behaviorally defined learning such as transfer, interference and spontaneous recovery. Finally, in order to be a practical working model, it should be reliable, robust and obtainable on demand at specific locations within the brain.

With these notions in mind, we have studied the phenomenon of kindling. The kindling effect is observed when stimulation, usually electrical, is delivered repeatedly under constant conditions to some part of the brain, and the response to that stimulation progressively increases to include epileptiform seizure and, eventually, behavioral convulsion (Goddard et al., 1969). We have focused our attention on the aspect of change, not seizure. From the point of view of a post-synaptic neuron, it should not matter whether the arrival of transmitter substance follows upon a change in the environment, electrical stimulation of an afferent neuron, or a seizure in some other part of the brain. The only things that should matter are the type of transmitter, the spatial, temporal and quantitative aspects of the arriving transmitter, and the ongoing context or activity in other afferents to the post-synaptic cell.

It is possible that the seizures associated with kindling are due to the abnormality of the initial input. That is, the electrical stimulation will cause a bizarre pattern of synchronous tetanic activation of many adja- 
cent cells which do not normally act together in such a fashion. After repetition, and kindling, other cells act together in an abnormal way which is a seizure. But this need not imply pathology in the method of change by which one event gives rise to the other event. A bizarre parent may have a bizarre child through an entirely normal act of procreation. This attitude is undoubtedly an oversimplification and should not go unchallenged. The advantage of the simplification is that it allows one to concentrate on the changes that occur during kindling, and search for an explanation without assuming that it must lie in the domain of epilepsy or seizure generators.

Later we will describe our current attempts to follow the lead of Bliss et al (Bliss and Lomo, 1973; Bliss and Gardner-Medwin, 1973) and study stimulation-induced alterations in a monosynaptic evoked potential. But first we will review the evidence obtained from standard kindling techniques to see if it has implications relevant to the normal process of engram formation. The kindling effect may not possess all of the above mentioned minimal conditions for a model engram, but many of them have been demonstrated, and we hope to point out those which await investigation.

A typical procedure for kindling (Burnham, 1971; Gaito, 1974; Goddard, 1967; Goddard et al., 1969; Morrell, 1973; Racine, 1972a, b; Tanaka, 1972; Tress \& Herberg, 1972; Wada \& Sato, 1974) is to apply electrical pulses or sine waves through a bipolar electrode implanted in the amygdala. The stimulation parameters are not critical provided that they involve fairly high frequency, usually above 25 $\mathrm{Hz}$, moderate amplitude, $50-100 \mu \mathrm{A}$ peak to peak in the rat (more in the cat and monkey), and a stimulus train duration of one or several seconds. The stimulation train is usually repeated under constant conditions once each day. More frequent application has been found to be less efficient in the sense that more trials are required to reach the first generalized behavioral convulsion. In the extreme case of stimulation applied continuously for many hours or days, behavioral convulsions are rarely observed (Goddard et al., 1969) except under special conditions (Racine et al., 1973) which may favor a form of recruiting response.

It is not critical that initial applications of the stimulation provoke local epileptiform after-discharge. Provided that the stimulation parameters exceed some undefined threshold, a local after-discharge will appear after several repetitions. Stated in other terms, the threshold for after-discharge is reduced by repeated application of sub-threshold stimulation (Racine, 1972a).

On additional trials the epileptiform after-discharge is observed to increase progressively in amplitude, duration and complexity of waveform, and propagates to more disparate areas of the brain (Goddard, 1972; Morrell, 1973; Racine, 1972b; Wada \& Sato, 1974). In association with the greater afterdischarge involvement, the behavior of the animal changes from arrest with slight facial contractions, blinking and masticatory seizures, to head nodding, partial opisthotonus, contralateral forelimb clonus, bilateral forelimb clonus, rearing and falling. In the fully kindled animal, when a convulsion is triggered, the onset latency is usually quite long (several sec. to $0.5 \mathrm{~min}$.) and the history of convulsion development tends to be recapitulated as the seizure builds (Goddard et al., 1969; Wada \& Sato, 1974). The exact form of the behavioral convulsion varies with species and strain; cats and monkeys typically show more severe and complex convulsions than the rat.

Between trials, a kindled animal appears to be quite normal, at least to casual observation and normal handling. Electrographic recording from the area of stimulation often reveals abnormal sporadic interictal spike discharge which, in the rat, is typically gone within 2 or 3 days after the last convulsion (Walters, 1970) but which in the cat may be permanent (Wada et al., 1974). Careful psychological testing of the animals reveals long lasting disruption of the ability to learn a conditioned emotional response in kindled rats (McIntyre \& Molino, 1972) or a reduction of predatory aggression in partially kindled cats (Adamec, 1975).

Examination of the site of stimulation with standard techniques for the light microscope or electron microscope has revealed no gross abnormality which cannot be observed near the tip of a control electrode in animals which were not kindled or did not receive any electrical stimulation (Goddard, 1972; Goddard \& McIntyre, 1974). Other control experiments indicate that the kindling effect results from neuronal activation and does not result from tissue damage, edema, gliosis or other time dependant biological processes that follow implantation of the electrode, nor from poison by metallic ions from the electrode or other by-products of the applied current (Goddard et al., 1969). Furthermore, Vosu and Wise (1975) have recently shown that kindling can result from repeated focal chemical stimulation. Injections of the cholinergic agonist carbachol were given once every second day through chronically implanted cannulas in the amygdala, hippocampus or caudate nucleus of the rat.

Both the chemical stimulation studies and the electrical stimulation studies indicate regional differences in responsiveness. In the rat, kindling is possible from anterior neocortex, olfactory bulb, olfactory area, preoptic area, pyriform cortex, amygdala, entorhinal cortex, hippocampus, septal area, caudate nucleus, globus pallidus and some areas of the thalamus. Within the limbic system, the most responsive area seems to be the amygdala (Goddard et al., 1969; Racine, 1972a, b; Vosu and Wise, 1975). Kindling does not seem to be possible by stimulation of posterior dorsal neocortex, much of the thalamus, midbrain reticular formation, red nucleus, substantia nigra or cerebellum (Goddard et al., 1969; Racine, 1972b, 1975). The hypothalamus may be a special case in which stimulation of the posterior lateral areas does not cause kindling whereas very strong stimulation of the medial and anterior areas can in- 
duce kindling, probably by indirect activation of the amygdala via anatomical connection from the site of stimulation (Cullen \& Goddard, 1975).

A behavioral convulsion can rarely be triggered on the first application of focal stimulation within the limbic system. The great difficulty, or impossibility, of triggering trialone convulsions by stimulating the amygdala, even at extremely high intensities (Goddard et al., 1969) is taken to indicate that epileptiform discharge in the amygdala is not able to drive a motor convulsion until the "connections" to the motor system have been strengthened or created by repetition. An exception to this rule is the aforementioned recruiting type of stimulation of Racine et al (1973), but even in this case the behavioral convulsion does not develop until the stimulation has been continued for one or several minutes. Thus the process of kindling involves more than threshold reduction in the amygdala, and implies the establishment of more widespread modification of brain function.

This implication of widespread change is strengthened by several other observations. Electrolytic destruction of all neurons which were stimulated directly during kindling does not prevent immediate triggering of the convulsion when a higher intensity current is passed through the lesion to activate neurons on the periphery of the lesion. Such stimulation of the periphery of a lesion in unkindled control rats does not cause convulsions until it has been repeated many times (Goddard et al., 1969). Repeated stimulation of a second area, following initial kindling, results in more rapid kindling than would otherwise be expected from the second area (transfer), even if the primary locus of kindling has been destroyed (Goddard et al., 1969; Racine, 1972b; Burnham, 1971). While bilateral activation of limbic structures can result in more rapid kindling, still several trials are required before motor convulsions appear (Racine et al., 1972b; Madryga et al., 1975). Electrographic recording shows that the midbrain reticular formation does not partici- pate in the seizure activity until late in the kindling process and yet is important for the modifications to follow (Wada \& Sato, 1975).

In addition to being quite widespread, the al terations in brain function that accompany kindling appear to be relatively permanent. Once an animal has been kindled to the point of behavioral convulsions, the convulsion can be triggered easily, even after several months without stimulation (Goddard et al., 1969; Wada et al., 1974). Furthermore, the behavioral alterations induced by kindling are long lasting. McIntyre and Molino (1972) observed poor learning of a conditioned emotional response even though the first training trial did not begin until 26 days after the last convulsion and was continued for 23 days after that. Adamec (1975) found interference with predatory aggression in some of his cats one month after only partial kindling. Thus, although the brain does not seem to be damaged by kindling, it is altered, perhaps permanently, and the alteration effects how the animal will respond to its environment.

Although an interval without stimulation does not attenuate the convulsive response to subsequent stimulation, there is one situation in which the convulsion can be lost. The loss, however, is only temporary. This is seen when kindling from a primary site is followed by secondary kindling from the amygdala. The primary site may be in some area of the limbic system and the secondary site in the ipsilateral amygdala. Or the primary site may be in one amygdala, and the secondary site in the contralateral amygdala. In each case the secondary site kindles more rapidly, as stated earlier (transfer). However, following secondary kindling from the amygdala, convulsions cannot be triggered from the original primary site (Goddard et al., 1969; Burnham, 1971; McIntyre \& Goddard, 1973). Convulsions can easily be re-kindled by primary site stimulation, but more importantly, a rest interval of one to two weeks will accomplish the same effect (McIntyre \& Goddard, 1973). That is, the primary site spontaneously recovers from the "suppression" when secondary site convulsions are discontinued.

These findings of transfer and retroactive interference bear at least superficial similarities to transfer and retroactive interference found in learning experiments. Proactive interference has also been shown to occur during kindling (McIntyre \& Goddard, 1973) by allowing a rest interval between primary kindling of one amygdala and secondary kindling of the contralateral amygdala. In this situation, the amount of transfer (faster secondary kindling) is greater following a rest interval of two weeks than without the rest interval. That is, primary kindling in addition to providing the long term basis for more rapid secondary kindling, also sets up a counteracting proactive interference to secondary kindling which spontaneously dissipates over a two week period.

Thus, in terms of the engram model, it is clear that the observation of kindling indicates a nondegenerative change in neural processing, which can result from neural activation, and which is relatively permanent. It occurs reliably in an adult mammalian brain, and is able to alter normal behavior in a lasting way. The phenomenon shows other similarities to behaviorally defined learning such as transfer, interference and spontaneous recovery (see also Gaito, 1974). As a practical working model it has proved to be reliable, robust, of large magnitude and obtainable on demand by stimulating specific locations within the brain. The particular locations from which kindling can be obtained are areas often considered important for motivation, reinforcement or learning.

Two basic properties remain to be demonstrated. The changes must be shown to be induceable by environmental sensory input, and to be selective enough in character to provide a theoretical basis for normal memory. To answer both of these problems it is first necessary to specify the exact locus and type of change in more detail.

One fact is clear. The change involves an increase in synaptic con- 
nectivity that is not restricted to seizure activity per se. Racine et al. (1972a) recorded potentials from the frontal pole, ventromedial hypothalamus, preoptic area and hippocampus that were evoked by brief test pulses delivered to the rat amygdala. The same electrode was used for delivery of the kindling stimulation and for delivery of the test pulses before and after kindling. Late components of the evoked potentials $(20-60 \mathrm{msec}$.) were significantly increased following the kindling trials, and remained at the increased level when tested 5 days later. These results indicate that synaptic conduction of neural activity is enhanced in a lasting way by the kindling process, and remains enhanced in the absence of ongoing seizures.

One approach to specifying the nature of the change would be to examine the brain directly for structural modification. A difficulty is that the locus of the change has not been adequately determined. For example, the Racine et al (1972) differences were observed in late components of the evoked potential, indicating a chain of several synapses between the stimulation and recording sites. Thus it is not clear precisely which synapses or cells were responsible for the observed potentiation. Perhaps one or perhaps all links in the chain were altered. We have attempted to study the morphology of the first link in the chain, namely the synapses at the axon terminals of amygdaloid neurons which were stimulated during kindling.

\section{METHOD}

Littermate male hooded rats of the Charles River Long-Evans pathogen free strain were randomly assigned into two groups of six. At age 62-65 days, all rats were implanted with electrodes in the basal nuclei of the amygdala and allowed to recover for 11 days. One group was kindled $(62.5 \mathrm{~Hz}, 1$ msec. diphasic pulses, 75 $\mu \mathrm{A}$ peak to peak for $2 \mathrm{sec}$. each day), the other group served as non-stimulated controls and were placed in the apparatus for an equal period of time each day. After the kindled animals had responded with 6 bilateral behavioral convulsions, all animals were maintained without stimulation for 7 days and the EEG was periodically sampled to ensure that abnormal activity (inter-ictal spikes) had disappeared. Thus, the experiment was designed to examine the synapses at a time after kindling when the ongoing activity had returned to normal and only the longlasting predisposition for the kindled seizure remained.

A small electrolytic lesion was then placed at the tip of the electrode in all rats to destroy the neurons in the area of stimulation, or control area, and "label" their axon terminals with degeneration. Two lesioned animals from each group were allowed to survive for either 1 , 2 or 4 days after the lesion before they were sacrificed. Age at sacrifice was 95-109 days.

Each rat was anesthetized with chloral hydrate and the EEG checked for possible abnormality (activation of the focus). The brain was fixed by perfusion with formaldehyde-gluteraldehyde in phosphate butter, removed and hand sliced into $0.5 \mathrm{~mm}$ slabs. The area containing the amygdala was saved from each of the relevant slabs and further fixed in formaldehydegluteraldehyde, washed, and postfixed with osmium tetroxide. The fixed blocks of tissue were embedded in araldite, oriented to permit sectioning vertically through the medio-lateral plane of the amygdala.

A $1 \mu$ section of the whole block face was stained with methelene blue and a drawing was made with the aid of a Leitz microscope drawing tube. The block was trimmed to leave a mesa of a chosen area protruding and the outline of another 1 $\mu$ section was added to the drawing. Silver sections from the area were mounted on bar grids and stained with both uranyl acetate and lead citrate for examination in a Phillips 200 electron microscope.

All possible degenerating terminals within an area bounded by two grid bars and the two ends of the tissue were recorded on $35 \mathrm{~mm}$ film at mag 12,200 . The positions of the grid micromanipulators were noted for each degenerating terminal and for the four corners of the tissue. In this way it was possible to enter the positions of all degenerating terminals on the original drawing of the block face.

The mesa was then shaved off the block, another drawing made of the whole block face, and the entire procedure was repeated. In this way, five or more samples could be obtained from up to five blocks from each amygdala, and serial drawings at approximately $50 \mu$ intervals made through the length of the amygdala. The series of drawings permitted accurate reconstruction of the size and location of the lesion at the electrode tip together with the locations of all observed degenerating terminals in the various amygdaloid nuclei (e.g. Fig. 1).

The electron micrographs were printed at a final mag 72,060 , and selected for adequate criteria of a morphological synapse associated

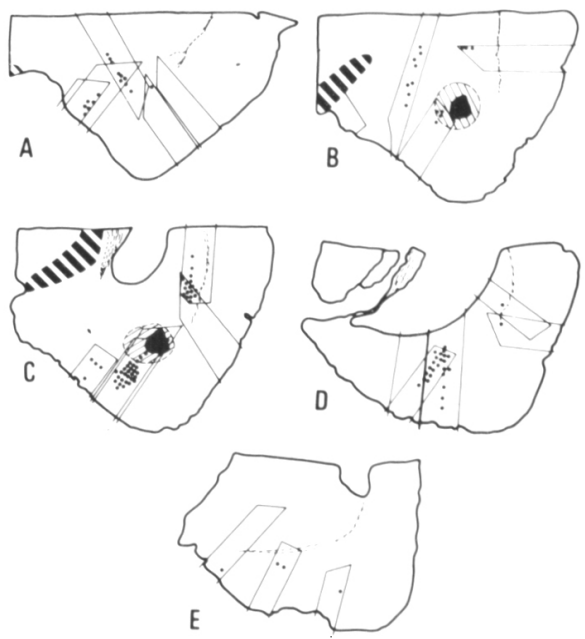

Figure 1-A-E: Composite drawings of 5 blocks of tissue taken serially through the amygdala of a kindled rat. A: rostral, E; caudal. Each drawing contains the outlines of the mesas (usually 5) that were made serially through the block of tissue. Dots indicate the locations of degenerating terminals found by electron microscopic examination of each mesa (all mesas were searched equally thoroughly). Black indicates the area of electrolytic coagulation used to destroy the neurons that had been stimulated during kindling. Light cross hatching indicates the area of partial damage (pale dendrites). Heavy cross hatching indicates optic tract. 
with the degenerating terminal (vesicle cluster, visible cleft, area of specialization in the apposed subsynaptic thickening). The selection was made blind, without reference to the source (amygdaloid nucleus, animal or group) and the prints were shuffled into random order and given a new set of code numbers to be doubly sure that the source of the terminal would not be known at the time of measurement.

Detailed measurements were made with a graphic to digital converter, accurate to .01 in. corresponding to $35 \AA$ in the tissue, and entered into a CDC 6400 computer for statistical analysis. The measures taken were: 1) length and breadth of the degenerating terminal, 2) width of two vesicles, 3) width of the cleft, 4) length and breadth of the post-synaptic structure e.g. spine, dendrite, 5) thickness of the sub-synaptic opacity, 7) length of the sub-synaptic opacity 8 ) length of the apposed membrane not covered by the sub-synaptic opacity 9) number of mitochondria in the degenerating terminal and 10 ) number of vacuoles in the degenerating terminal. In addition, the following qualitative descriptors were entered: 1) degree of confidence in those measures which vary with angle of sections, e.g. synaptic cleft, 2) vesicle shape: round, flat or pleiomorphic, 3) type of post-synaptic structure: spine, dendritic shaft, soma, axon, 4) presence or absence of a spine apparatus and 5) whether the sub-synaptic opacity consisted of one continuous strip or of several shorter strips.

The series was then decoded and information was added regarding the anatomical location of the terminal, number of days from lesion to time of sacrifice, whether or not the animal had been kindled, and the proportion of each of the amygdaloid nuclei that was damaged by the lesion at the electrode tip.

\section{RESULTS AND DISCUSSION}

A total of 1069 degenerating terminals of acceptable quality were measured, examples are shown in Fig. 2. Extensive statistical analyses were made for the groups overall and for various sub-groupings based on anatomical locations of the terminals, vesicle shape, size of the terminals, type of post-synaptic structure, etc. The rather surprising result was the paucity of consistent significant differences between kindled and control animals on any of the measures. The only noteworthy exception was that the identified terminals were consistently larger in kindled animals than in control animals. This difference proved to be confined to terminals containing pleiomorphic vesicles and not terminals containing round or flat vesicles. The largest number of terminals suitable for this analysis were located in the cortical nucleus of the amygdala. However, even this difference cannot be accepted without additional evidence since an analysis of covariance, in which the lesion location was used as a predictor, revealed that for this measure the lesions were not adequately matched between the kindled and control animals. The anatomical difference accounted for enough of the effect to render the kindled-control differences non-significant. In other words, the difference in the size of axon terminals could be due to kindling, or it could be due to differences in the anatomical locations of the parent cells, or it could be due to a combination of both factors. The available data do not permit a decision between these three alternatives.

However, it is clear that no gross abnormality is found in the synaptic morphology of neurons that were stimulated during kindling and that any differences which may result from kindling are quite subtle. In particular, electron-microscopic differences in the post-synaptic elements are unlikely since the visualization of these elements in the present study was good, not being obscured by the pre-synaptic degeneration that was used to identify the "kindled"' synapses.

The amygdala is perhaps the best structure from which to induce kindling, but it is not well suited for morphological experiments. The amygdala is anatomically heterogeneous and its connections, both intrinsic and extrinsic are complex. The above difficulties in attributing effects due to kindling and those due to anatomical location of the parent cells were due, in part, to this complexity. A more suitable structure would be the hippocampus where the cells and their connections are stratified, stereotyped and far more orderly. This anatomical orderliness not only simplifies morphological experiments, but greatly facilitates electrophysiological recording of mono-synpatic evoked potentials which can permit a more adequate determination of the locus of changes induced by kindling. Thus, before investigating morphology, it is possible to demonstrate kindling-induced alterations in the evoked potentials and examine those details that are relevant to altered synaptic physiology.

The field potential of interest here is that induced in the dentate granule cells by stimulation of the fibers of the perforant path, which arises in the entorhinal cortex. This is a mono-synaptic connection, and the orderly arrangement of cells in this area permits accurate identification of presynaptic potential, excitatory post-synaptic potential (EPSP) and spike discharge, from field potentials recorded with a fairly large electrode (Lømo, 1971). Thus, measurements can be repeated reliably over long periods of time that would not be possible with intracellular recording. Bliss et al. (Bliss \& L $\varnothing \mathrm{mo}, 1973$; Bliss and Gardner-Medwin, 1973) have shown that this evoked potential can be potentiated by tetanic stimulation of the perforant path and that the observed potentiation may be very long lasting. Not all experiments showed the effect, but in one case the EPSP was still potentiated several weeks after the tetanization.

We have repeated the Bliss et al. observations in rats, with a modified method, and have obtained reliable long lasting potentiation (Douglas and Goddard, 1975). It is interesting that the tetanization stimulus used by Bliss et al., and by us, is very similar to the adequate stimulus for kindling. In one case where the stimulation was repeated at one trial per day, the animal eventually re- 


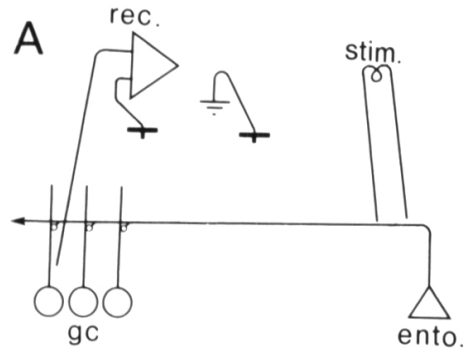

B

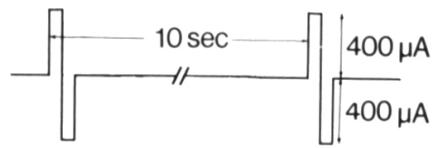

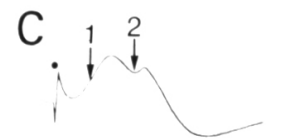
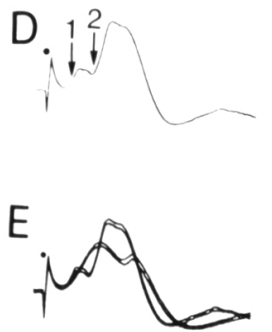

Figure 3-Hippocampal evoked potentials in a squirrel monkey. A; electrode configuration, rec: monopolar electrode among granule cells (gc) of fascia dentata referred to an indifferent screw electrode in the skull, stim: bipolar stimulation electrode among axons of the perforant path which originate in the entorhinal cortex (ento) and make synapses onto the dendrites of gc. B: the standard test train consisted of diphasic 400 \& A $100 \mu \mathrm{sec}$. pulse pairs delivered at $0.1 \mathrm{~Hz}$. C, D: examples of the averaged evoked potential produced by the standard test train before any high frequency stimulation $(C)$ and after kindling (D). Symbols: $\bullet$ stimulus artifact, $\downarrow^{1}$ positive deflection indicating the rising leg of the extracellular field excitatory post-synaptic potential (EPSP), $\downarrow^{2}$ negative deflection indicating cell discharge. $\mathrm{E}$ : the $95 \%$ confidence limits of $C$ and of $D$, wherever the two sets of confidence limits do not overlap indicates a statistically significant difference between the waveforms. Note the differences in rise time of 1 , latency and amplitude of 2 and overall amplitude of peak positivity (up).
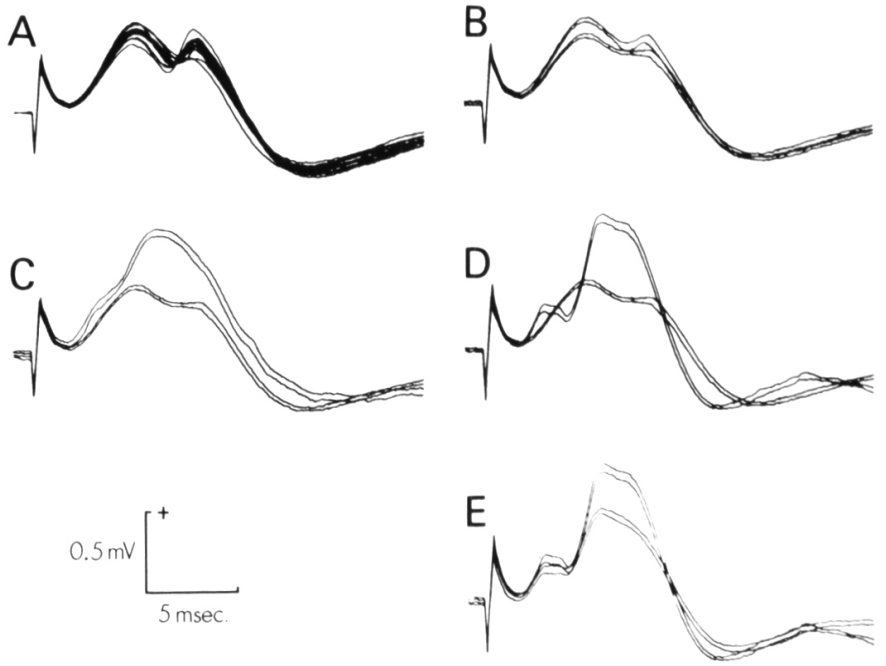

Figure 4-Waveforms recorded as in Fig. 3 at different times of the experiment. All waveforms were photocopied directly from computer display. A: baseline potentials averaged each day for 14 days and superimposed. B: cumulative result of subthreshold high frequency stimulation trains showing significant reduction in amplitude (day 22 compared to day 15). C: result of a single kindling trial, $24 \mathrm{hr}$. after stimulation, showing significant potentiation of the EPSP (day 23 compared to day 22). D: result of 10 kindling trials, $24 \mathrm{hr}$. after most recent stimulation, showing significant potentiation of EPSP, population spike and population spike latency (day 39 compared to day 22). E: attenuation of the potentiation by a 10 day rest interval, showing that some components of the waveform were significantly smaller (day 51 compared to day 39). But note that the final waveform had not reverted back to the pre-kindling or baseline levels. In particular, the population spike latency maintained its post-kindling value. sponded with behavioral convulsions. As in kindling, however, it was not necessary for the tetanization stimulus to induce an afterdischarge on trial-one for potentiation of the EPSP to be observed, or for after-discharge to appear on subsequent trials.

\section{METHOD \& RESULTS}

We are now investigating this effect in the squirrel monkey. An example is presented in Figs. 3 and 4. The electrodes were implanted under Nembutal anesthesia, stereotaxic guidance and continuous monitoring of the evoked potentials. A tungsten bipolar electrode, $2 \mathrm{~mm}$ tip separation, was located in fibers of the perforant path in the left hemisphere, a tungsten monopolar recording electrode was located in the ipsilateral dentate area, an indifferent screw electrode was placed in the skull over the frontal cortex, and another, contralateral, screw electrode was used for ground (Fig 3A). The leads for all electrodes were connected to a miniature plug and all components were fixed in place with skull screws and dental acrylic. The monkey was allowed two months to recover from surgery.

The standard test train was arbitrarily set at diphasic pulse pairs, each of $0.1 \mathrm{msec}$. and $400 \mu \mathrm{A}$, repeated 10 times at one per $10 \mathrm{sec}$. (Fig 3B) and held constant throughout the study. The recorded potentials were preamplified (Grass P15B), amplified (Tektronix $5 A 18 N$ ), and sampled with a PDP-11 computer at a rate of one point per $45 \mu \mathrm{sec}$. Each point was averaged for the 10 sweeps each day. In addition, the standard error of each point mean was computed to permit display of the $95 \%$ confidence limits and statistical comparisons at each time point in the waveform between difference days of the experiment (Fig 3E). Initially the standard test train evoked a potential in which the field EPSP and a small negative deflection indicating cell discharge could be seen (Fig. 3C).

The unmodified potential was recorded each day for two weeks (Fig. $4 \mathrm{~A})$. Then a series of subthreshold high frequency stimulation trains were delivered in the course of the following week. These subthreshold trains included bursts of standard pulses delivered at $400 \mathrm{~Hz}$ for 25

Figure 2-Examples of degenerating terminals found in six different rats. $C$ : control animals, no electrical stimulation prior to lesion at electrode tip. K: kindled animals with similar lesion at electrode tip. 1, 2, 4: days of survival between lesion and perfusion. The examples selected all make an asymmetrical synapse with a dendritic spine. 


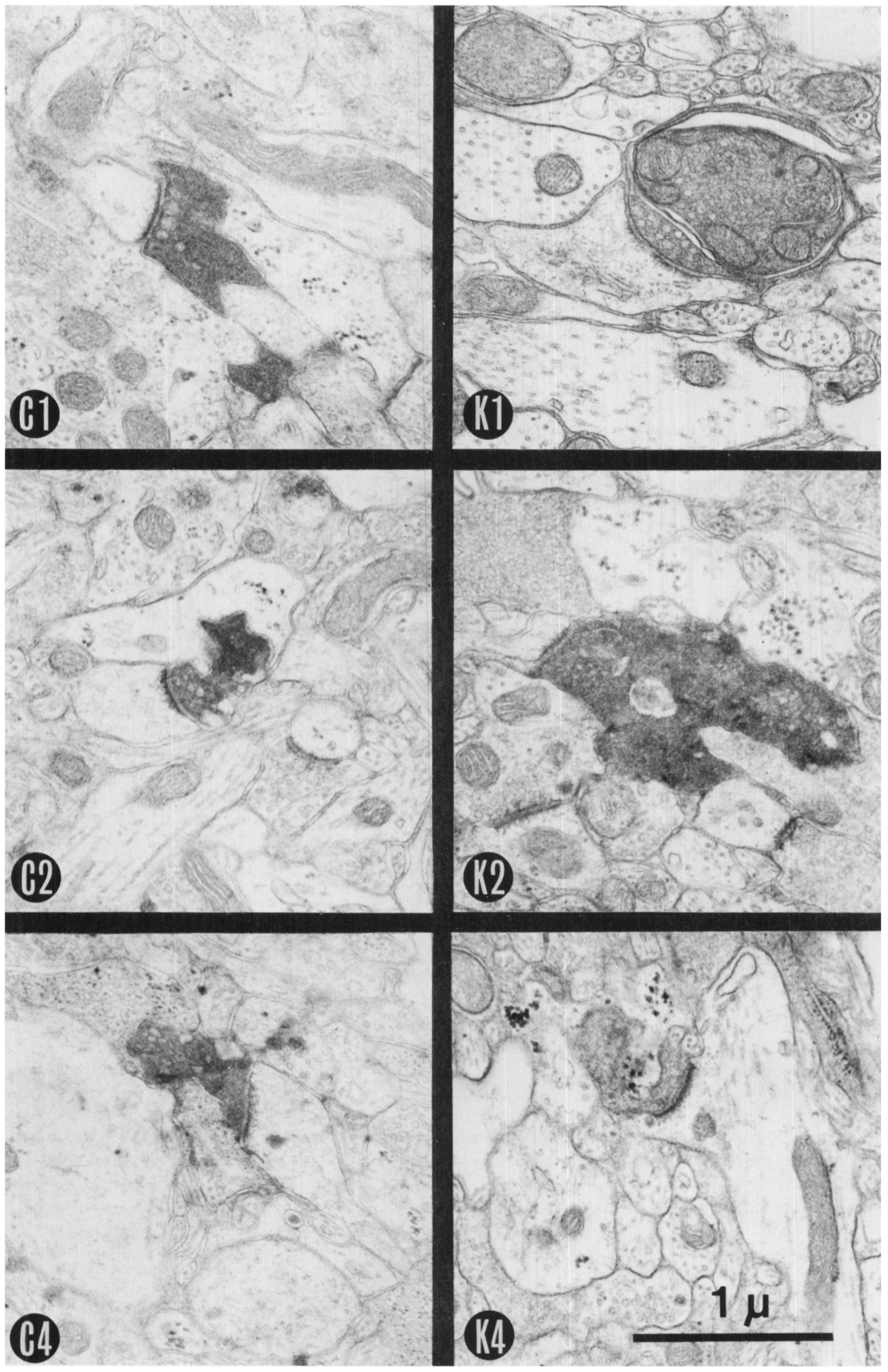


msec. and repeated at one burst per $10 \mathrm{sec}$. The total number of bursts was either 10 or 20 and, on one occasion included a series in which the initial pulse was of double amplitude and double duration. Then one series was presented at a repetition rate of one burst per sec. A train of standard pulses was finally delivered at $10 \mathrm{~Hz}$ for $7 \mathrm{sec}$. These trains have been shown to produce good potentiation in the rat (Douglas, unpublished observation) so there may be a species difference in threshold for potentiation.

Twenty-four hours after each of the subthreshold trains, the evoked potential appeared to be slightly reduced. The cumulative amount of this reduction is shown in Fig. 4B. It can be seen that the confidence intervals during the EPSP component do not overlap, indicating a significant attenuation of the EPSP from day 15 , the day before the series of subtreshold stimulation trials, to day 22 , the day after the last subthreshold trial.

Then, on day 22 , the first kindling trial was delivered. This consisted of a $5 \mathrm{sec}$. train of standard pulses delivered at a repetition rate of $62.5 \mathrm{~Hz}$. The hippocampal EEG was monitored and revealed a $4 \mathrm{sec}$. high frequency epileptiform after-discharge. Twenty-four hr. later, day 23 , the EPSP was significantly potentiated (Fig. 4C) and an early population spike component (cell discharge) through very small in amplitude, was observed as a deflection in the rising leg of the EPSP (latency $4.5 \mathrm{msec}$.)

Standard test trains were continued once each day and the kindling stimulus was repeated 10 times. This amount of kindling was sufficient to increase the after-discharge duration to $7.5 \mathrm{sec}$., but was not sufficient to involve behavioral manifestations of the seizure activity. Fig. 4D shows that the extra kindling was accompanied by a small additioned increase in the EPSP and an increase in the size of the population spike which remained at a peak latency of $4.5 \mathrm{msec}$.

Only two standard test trains were administered in the following 10 day interval, and this rest interval was accompanied by a reduction in amp- litude of the EPSP and a reduction in amplitude but no change in latency of the population spike (Fig. 4E). The rest interval, however, was not sufficient to reduce the potential to levels observed prior to the kindling trials. The final waveform showed faster rise time of the EPSP, higher amplitude of EPSP, and shorter latency of the population spike. These differences were all larger than could be evoked in a pre-kindling test using test pulses of double amplitude and double pulse width. It should be recognized, however, that a 3.4 msec. shift in latency of the cell discharge observed by recording field potentials need not imply that each individual cell alters its spike latency by this amount.

\section{DISCUSSION}

These results from the squirrel monkey are consistent with those obtained in the rat (Douglas and Goddard, 1975) and the rabbit (Bliss and Lømo, 1973: Bliss and Gardner-Medwin, 1973), where control experiments and measurement of the pre-synaptic fiber potential suggest that the potentiation is a synaptic phenomenon based on either the release of more transmitter from the axon terminals or lasting sensitization of the post-synaptic membrane.

Thus it would appear that kindling is caused, in part, by a lasting potentiation of excitary synapses. More work is needed to decide whether the changes are pre-synaptic or in the post-synaptic membrane, whether they are accompanied by alteration in synaptic morphology, whether they can occur in other or all types of excitatory and inhibitory synapses, whether the response of the post-synaptic cell is of any importance and, in general, what distinguishes a subthreshold stimulus from a kindling stimulus. Answers to all of these questions are vital to the issue of what constitutes an engram and whether the artificial engram of kindling is a relevant model for the engram of normal memory.

However, it should be stressed that the engram issue is only one aspect of kindling. We have chosen to concentrate on this one aspect of kindling, to the exclusion of all others. We believe that this form of tunnel vision has led to some interesting observations on kindling that might otherwise have gone unnoticed. And we hope that the research will provide some insight into why kindling, and possibly other forms of focal epilepsy, are both progressive and very durable.

Clearly, an adequate description of epilepsy must also include proper emphasis on the nature of epileptiform activity and the method by which epileptiform activity spreads from a focus to engage other areas of the brain. It is very likely that lasting potentiation of the relevant synapses would aid in the propagation of epileptiform activity. The phenomenon of kindling cannot be explained entirely by a process of synaptic potentiation. Both the latency, in the order of seconds, between onset of stimulation and appearance of the behavioral convulsion, and the stereotyped pattern of the convulsion itself suggest that considerable processing of the abnormal activity occurs before the behavioral convulsion is initiated. Many factors other than direct linear synaptic excitation must be involved. Neural or endocrine feedback, collapse of inhibition, temporary imbalance of extracellular ionic concentrations or energy mechanisms might all play some role. We have chosen to concentrate on only the one aspect, permanent change and the engram issue.

Our answer to the question: does the engram of kindling model the engram of normal long term memory?, is yes, kindling does have many properties expected of a model engram. It involves a non-degenerative change in neural processing based, at least in part, on relatively permanent alterations of excitatory synapses. It can be induced by specific forms of neural activation at specifiable locations in the adult mammalian brain, and can affect behavior in a lasting way. And, the effect shows other similarities to learning such as transfer, interference and spontaneous recovery. However, it is not yet known if similar changes are induced in the adult brain by environmental sensory input, and more 
details of the precise nature of the kindled synaptic modification, together with a better knowledge of the rules controlling the modification, are needed before it will be clear if the effect could be the same as the agent of normal memory.

It must always be understood that we are dealing here with an analogy. This type of research can never prove that the empirical model involves the same physiological mechanism as that used during normal memory formation. All it does is demonstrate whether the assumptions specified by theories of memory describe events that can and do occur in a real brain. Thus the data may be used to select between competing theories. The real hope for this type of work is that the observations will provide new insight into a conceptual framework that leads to a revised and more complete theory.

\section{DISCUSSION}

Dr. Racine: Could you comment on the adequacy of the "pre-synaptic component" as a measure of output from your stimulated site? Some electrophysiologists might question the sensitivity of the field potential recording technique to changes in the intensity of the pre-synaptic component compared to changes in the post-synaptic component, particularly in view of the fact that pre-synaptic activity is fast and occurs within small terminal fibers and boutons. Dr. Goddard: In our work on the rat, where we have had some electrodes that recorded a good pre-synaptic potential, we have never seen a measurable increase that might account for the increased EPSP. It is possible that the pre-synaptic change, if one exists, is too small for us to detect and yet large enough to account for the obviously larger post-synaptic components. We are currently trying to improve our technique in two ways. First, we are measuring orthodromically both the pre- and post-synaptic potentials evoked by different amplitudes of stimulation. This should allow us to see how much the EPSP increases when there is a small but measurable increase in the pre-synaptic component. By inference, we may be able to decide whether the increase in EPSP seen following kindling is within the range of that normally accompanying measurable changes in the pre-synaptic component. Secondly, we are trying to record the antidromic spike in the entorhinal cortex. In exceptionally clear examples, it may be possible to compare any growth of the orthodromic to that of the antidromic component. Such a difference may reflect the contribution of depolarization in the axon terminals. If axon terminals become larger following kindling, the effect may be revealed by such measures. We do not have data to report at this time. Dr. Racine: would like to share our knowledge of some control problems that we have encountered in our own potentiation work that might apply to your "perforant path" preparation. We find that the amplitude and wave form of a number of different types of evoked responses, including responses evoked in other brain areas by stimulation of the cortex or hippocampus, show a very close correlation with motor behavior. The transcortical potential, for example, increases in amplitude when the animal is relatively motionless compared to when the animal is walking. These correlations with behavior are similar to those reported by Vanderwolf for the spontaneous patterns of activity within the hippocampus. The stimulation treatment we use is often accompanied by a change in motor behavior during the course of an experiment. I have no idea whether or not the perforant path system shows the same sensitivity to changes in behavior. Do you have any information about this? Dr. Goddard: We have not made a careful study of the perforant path granule cell potential in correlation with particular types of motor behavior. But we have sampled the potential at regular $20-30 \mathrm{~min}$ intervals for periods of 24 or 48 hours in freely moving rats. The potentials fluctuate within the time span of one average $(100 \mathrm{sec})$, they fluctuate between averages, and they fluctuate with a small but quite distinct circadian rhythm. The baseline also fluctuates when the animal is tested repeatedly at the same time of day over a period of many days. However, the amplitude of all these fluctuations is an order of magnitude smaller than the sudden alteration that is seen following kindling. The example from the squirrel monkey, shown here, was taken while the animal was sitting in a primate chair. The behavior was observed on all occasions, but no behavioral change was noticed. Dr. Burnham: Isn't the "anterior limbic field" really part of the motor cortex? Dr. Goddard: At the time we published our results on anterior cortex we tried to make sense out of them in terms of the motor cortex. The relation was not simple. We used the term "anterior limbic field" with some reluctance. finding no better term to encompass the data. Most certainly the question should be reexamined, but we have not pursued it ourseIves. Dr. Burnham: Do you consider "interference" as a learning analogue? I know you thought of it as active trace competition circa 1969, but these days doesn't the evidence suggest that it's a non-specific, nonpermanent generalized alteration of excitability? Dr. Goddard: Yes, the evidence at the moment suggests a very widespread interference or suppression of epileptiform activity that affects all sites tested except the one from which the convulsions were most recently triggered. It decays spontaneously in the time span of about two weeks. The difficulty of relating this effect to other physiological or psychological phenomena is that very few phenomena have been studied in this time domain. It would be foolish to place much emphasis on this aspect until much more is known, but equally. I would not reject the possibility that it is related to important and normal psychophysiological events. Dr. Morrell: It seems to me very important that kindling is not simply a change in the threshold to stimulation. It is not an alteration of excitability. It is the establishment of a particular and specific circuit in the brain in which there are alterations of excitability parameters, but it is the specificity of the circuit which I believe is really the critical dimension. In many of the kindling studies, probably all of you have noted, although there haven't been very many publications about it, there are in the electrical records in between the intervals of stimulation, spontaneous paroxysmal discharges. These have a very interesting characteristic. Like the evoked potential, the population response - we call these spontaneous hypersynchronous potentials or SHP's - the configuration of the electrical response is the function of the site and sequence of synaptic activation in the population which generates it. Here are some hypersynchronous potentials in a preparation which I will discuss in my report a little later, but what 1 want to illustrate is that here are these spontaneous events occurring now in interstimulus intervals and, in this section, is a single one occurring in the primary and secondary hemispheres. Look now at the primary hemisphere and we have simply a superimposed tracing of the next five hypersynchronous potentials in that sequence and the superimposition is remarkable. You can go on and do that indefinitely and the coherence in the wave shape of those potentials is striking. Here is another example and in the superimposed traces if you look carefully, you can even see that the very fine, fast transients are reproduced in the superimposed traces. I am sure that if one did computer coherence analysis one would find a very substantial degree of coherence. Not only does this apply to the spontaneous potential, say, on the side kindled but eventually the involvement of the contralateral hemisphere occurs also, even if the primary hemisphere is not necessarily spontaneous but is triggered by discharge in the secondary hemisphere and yet the wave shape elicited is exactly the same. Again this suggests that what has happened is that a population has a certain anatomical and physiological regularity and specificity in the sense of sequence of synaptic activations.

\section{REFERENCES}

ADAMEC, R. (1975). Behavioral and epileptic determinants of predatory attack behavior in the cat. The Canadian Journal of Neurological Sciences.

BLISS, T. V. P. and LØMO, T. (1973). Longlasting potentiation of synaptic transmission in the dentate area of the anaesthetized rabbit following stimulation of the perforant path. Journal of Physiology, 232, 311-356.

BLISS, T. V. P. and GARDNER-MEDWIN. A. R. (1973). Long-lasting potentiation of synaptic transmission in the dentate area of the unanaesthetized rabbit following stimulation of the perforant path. Journal of Physiology, 232, 357-374.

BURNHAM, W. M. (1971). Epileptogenic modification of the rat forebrain by direct and trans-synaptic stimulation. Doctoral dissertation, McGill University, Montreal.

CULLEN, N. and GODDARD, G. V. (1975) Kindling in the hypothalamus and transfer to the ipsilateral amygdala. Behavioral Biology, in press.

DOUGLAS, R. M. and GODDARD, G. V. (1975). Long-term potentiation of the perforant path-granule cell synapse in the rat hippocampus, Brain Research, 86, 205-215. 
GODDARD, G. V. (1967). Development of epileptic seizures through brain stimulation at low intensity. Nature, 214, 1020-1021.

GODDARD, G. V. (1972). Long term alteration following amygdaloid stimulation. In B. Eleftherion (Ed.), The Neurobiology of the Amygdala. Plenum Press, New York, 581-596.

GODDARD, G. V. and McINTYRE, D. C. (1974). Some properties of a lasting epileptogenic trace kindled by repeated electrical stimulation of the amygdala in mammals. In L. V. Laitinen and K. E. Livingston (Eds.). Surgical Approaches in Psychiatry, University Park Press, Baltimore, 109-115.

GODDARD, G. V., McINTYRE, D. C. and LEECH, C. K. (1969). A permanent change in brain function resulting from daily electrical stimulation. Experimental Neurology, 25, 295-330.

GAITO, J. (1974). The kindling effect. Physiological Psychology, 2, 45-50.

HEBB, D. O. (1949). The Organization of Behavior, a Neuropsychological Theory. John Wiley \& Sons, New York.

LASHLEY, K. S. (1950). In search of the engram. In Society of Experimental Biology Symposium No. 4. Physiological Mechanisms in Animal Behavior. Cambridge University Press, 454-482.

L $\emptyset_{M O}, T$. (1971). Patterns of activation in a monosynaptic cortical pathway: the perforant path input to the dentate area of the hippocampal formation. Experimental Brain Research, 12, 18-45.

McINTYRE, D. C. and GODDARD, G. V. (1973). Transfer, interference and spontaneous recovery of convulsions kindled from the rat amygdala. Electroencephalog- raphy and Clinical Neurophysiology, 35, 533-543.

McINTYRE, D. C. and MOLINO, A. (1972). Amygdala lesions and CER learning - long term effect of kindling. Physiology and Behavior, 8, 1055-1058.

MADRYGA, F. J., GODDARD, G. V. and RASMUSSON, D. D. (1975). The kindling of motor seizures from hippocampal commissure in the rat. Physiological Psychology, in press.

MARR, D. (1969). A theory of cerebellar cortex. Journal of Physiology, 202, 437-470.

MILNER, P. M. (1957). The cell assembly: Mark II. Psychological Review, 64, 242-252.

MORRELL, F. (1973), Goddard's kindling phenomenon: a new model of the "mirror focus." In H. C. Sabelli (Ed.) Chemical Modulation of Brain Function. Raven Press, New York, 207-223.

RACINE, R. J. (1972a). Modification of seizure activity by electrical stimulation: $I$. After-discharge threshold. Electroencephalography and Clinical Neurophysiology, 32,269-279.

RACINE, R. J. (1972b). Modification of seizure activity by electrical stimulation: II. Motor seizure. Electroencephalography and Clinical Neurophysiology, 32, 281-294.

RACINE, R. J. (1975). Modification of seizure activity by electrical stimulation: Cortical areas. Electroencephalography and Clinical Neurophysiology, 38, 1-2.

RACINE, R. J., BURNHAM, W. M. and GARTNER, J. C. (1973). First trial motor seizures triggered by amygdaloid stimulation in the rat. Electroencephalography and Clinical Neurophysiology, 35, 487-494.
RACINE, R. J., GARTNER, J. G. and BURNHAM, W. M. (1972a). Epileptiform activity and neural plasticity in limbic structures. Brain Research 47, 262-268.

RACINE, R., OKUJAVA, V. and CHIPASHVILI, S. (1972b). Modification of seizure activity by electrical stimulation: III. Mechanisms. Electroencephalography and Clinical Neurophysiology, 32, 295-299.

TANAKA, A. (1972). Progressive changes of behavioral and electroencephalographic responses to daily amygdaloid stimulations in rabbits. Fukuoka Acta Medica, 63, 152-164.

TRESS, K. H. and HERBERG, L. J. (1972). Permanent reduction in seizure threshold resulting from repeated electrical stimulation. Experimental Neurology, 37, 247-359.

VOSU, H. and WISE, R. A. (1975). Cholinergic seizure kindling in the rat: Comparison of caudate, amygdala and hippocampus. Behavioral Biology, in press.

WADA, J. A. and SATO, M. (1974). Generalized convulsive seizure induced by daily electrical stimulation of the amygdala in cats: Correlative electrographic and behavioral features. Neurology, 24, 565-574.

WADA, J. A. and SATO, M. (1975). The generalized convulsive seizure state induced by daily electrical stimulation of the amygdala in split brain cats. Epilepsia, in press.

WADA, J. A., SATO, M. and CORCORAN, M. E. (1974). Persistant seizure susceptibility and recurrent spontaneous seizures in kindled cats. Epilepsia, 15, 465-478.

WALTERS, D. J. (1970). Sporadic Interictal Discharges in Kindled Epileptogenic Foci. Unpublished M.A. Thesis. Dalhousie University. 